\title{
In silico broadband mechanical spectroscopy of amorphous tantala
}

\author{
F. Puosi $\odot,{ }^{*}$ F. Fidecaro $\odot$, S. Capaccioli $\odot$, D. Pisignano $\odot$, and D. Leporini $\odot^{*}$ \\ Dipartimento di Fisica “Enrico Fermi,” Università di Pisa, Largo B. Pontecorvo 3, I-56127 Pisa, Italy \\ and Istituto Nazionale di Fisica Nucleare, Largo B. Pontecorvo 3, I-56127 Pisa, Italy
}

(Received 7 June 2019; published 21 November 2019)

\begin{abstract}
Adopting an agnostic approach, the quality factor $Q$ of tantala glass is drawn via in silico mechanical spectroscopy in wide ranges of temperature $(10-300 \mathrm{~K})$ and frequency $(500 \mathrm{MHz} \leqslant f \leqslant 1 \mathrm{THz})$. At the highest frequencies, $Q \propto f^{-3}$, consistent with Rayleigh sound scattering. For frequencies lower than terahertz, losses exhibit a weak power-law frequency dependence $Q \propto f^{-\alpha}$ with $\alpha \sim 0.1$ to 0.2 , depending on glass preparation and temperature. Arguing the validity of the power law down to $1 \mathrm{kHz}$, we show striking agreement with the losses measured in annealed amorphous films in the whole temperature range, revealing similitude between disordered structures created by different routes (quench cooling and deposition). Our results do not support the scenario of the mechanical loss due to activated relaxation of independent two-level systems.
\end{abstract}

DOI: 10.1103/PhysRevResearch.1.033121

\section{INTRODUCTION}

Recently, an increasing number of fundamental and applicative research fields faced limitations owing to the mechanical thermal noise: optomechanics [1-3] with nanomechanical thermal fluctuations [4], micro- and nanomechanical devices [3,5-7], nanosystems with high mechanical quality factor $Q$ of interest for radio astronomy, medical imaging, navigation, wireless communication [8], optical atomic clocks [9], gyroscopes [10], and optical components of gravitational wave detectors [11-14].

Mechanical thermal noise arises from damping mechanisms inside the material [15]. In particular, damping in disordered solids involve diverse phenomena, depending on the temperature and the frequency range of interest. We review the different mechanisms limiting our attention to temperatures above $\sim 10 \mathrm{~K}$ where quantum tunneling is negligible [16] and focus on frequencies $f$ below the Ioffe-Regel limit, i.e., below a few terahertz and $Q>\pi$ where the wavelength of the sound waves with attenuation factor $\Gamma=2 \pi f / Q$ is larger than the average interparticle distance and the notion of a phonon with a well-defined wave vector is applicable [17]. In the range of $\sim 0.4-1 \mathrm{THz}$, sound attenuation is due to the Rayleigh elastic scattering in a statistically disordered medium, leading to a contribution to the quality factor given by [17-21]

$$
\frac{1}{Q_{\text {Rayleigh }}(f)} \propto f^{3} .
$$

At lower frequencies, anelastic effects become apparent. One damping mechanism is ascribed to the entropy generated by the nonreversible heat flow inside the solid [7,22]. Given the

\footnotetext{
*francesco.puosi@df.unipi.it; dino.leporini@unipi.it

Published by the American Physical Society under the terms of the Creative Commons Attribution 4.0 International license. Further distribution of this work must maintain attribution to the author(s) and the published article's title, journal citation, and DOI.
}

fundamental large-scale character, the latter mechanism is observed in both crystals and disordered solids. Two heatflow mechanisms are known. In Akhiezer damping (AD), losses follow by the redistribution of the energy of the sound wave between the normal modes of the solid due to anharmonicity [7,18,20,23,24]. In contrast, in thermoelastic damping (TED), the thermal relaxation happens between straininduced temperature differences in distinct spatial areas of a solid $[7,22,25]$. The AD and TED contributions to the quality factor are encompassed by the simple expression,

$$
\frac{1}{Q_{i}(f)}=\Delta_{i} \frac{2 \pi f \tau_{i}}{1+\left(2 \pi f \tau_{i}\right)^{2}}, \quad i=\mathrm{AD}, \mathrm{TED}
$$

where both the strength $\Delta_{i}$ and the relaxation times $\tau_{i}$ depend on intrinsic thermodynamic properties of the material and, in the case of TED, also on the size and the shape of the sample if transverse elastic waves are excited [7,22,25]. Below $\sim 100 \mathrm{GHz}$, mechanical losses are also affected by internal friction which exhibit a much weaker frequency dependence of the related contribution to the quality factor with respect to the other ones, Eqs. (1) and (2) [20,21,26-30], exploited in rather popular models where the $Q$ factor is taken as frequency independent [31].

The mechanism of friction losses strongly depends on microscopic details, e.g., the irreversible motion of atoms during vibration in intrinsic [20,21] or extrinsic [32,33] defective zones localized in the bulk [2,3,7] or surface [32-34] of the material. Friction losses are usually described by a phenomenological approach where defects are modeled as two-level systems (TLSs) with a characteristic energy barrier $V$ and an asymmetry $\Delta$ between the two states [16,35]. Above $\sim 10 \mathrm{~K}$, tunneling across the barrier becomes less efficient than the thermally activated relaxations process (TARP). Accordingly, the relaxation time is given by the usual Arrhenius law $\tau=\tau_{0} \exp \left(V / k_{B} T\right)$ where $k_{B}$ and $\tau_{0}$, respectively, are the Boltzmann constant and a microscopic time on the order of $\sim 0.1$ ps [16,20,24,35-37]. Routinely, the TARP contribution 
to the quality factor is evaluated as [35]

$$
\begin{aligned}
\frac{1}{Q_{\mathrm{TARP}}(f)}= & \frac{\gamma^{2}}{k_{B} T C} \int_{0}^{\infty} \int_{0}^{\infty} \frac{2 \pi f \tau}{1+(2 \pi f \tau)^{2}} \operatorname{sech}^{2}\left(\frac{\Delta}{2 k_{B} T}\right) \\
& \times p(\Delta, V) d \Delta d V
\end{aligned}
$$

where $C$ is the appropriate elastic constant, $\gamma$ is the coupling between the defect and strain, and $p(\Delta, V)$ accounts for the distribution of TLS parameters. Equation (3) builds on several delicate assumptions: (i) Defects are taken as independent, despite a slowly decaying dipolar elastic interaction [38-40], for a review, see Ref. [37], (ii) the coupling between the sound wave and the TLS is rationalized in terms of homogeneous affine elasticity even if it is known that disorder leads to strong heterogeneous nonaffine strain [41-44], (iii) the microscopic nature of the TLS excitations, which is still unclear, is pictured in terms of $p(\Delta, V)$, taken as temperature independentwith unknown influence of how the amorphous structure is prepared (thermal history) - and, for simplicity, factorized in two terms $p(\Delta, V)=p(\Delta) p(V)$, invoking independence of the two variables. No general consensus has been reached on the most effective form of both $p(\Delta)$ and $p(V)$. In particular, $p(V)$ has a complex pattern [26] which is approximated as Gaussian [20], exponential [35,36], or with other forms [24].

The previous discussion suggests to focus on alternative agnostic methods to: (i) Evaluate the mechanical losses in disordered solids with no reliance on models and hardly verifiable assumptions, and (ii) assess the TARP scheme. To this aim, it seems proper to consider systems with low losses where the anticipated corresponding low density of defects better matches the assumptions of the TARP scheme. In this respect, amorphous solids are of interest [26,45], in particular, prototypical oxides, such as silica $[21,26,46]$, titania, and tantala. More specifically, amorphous tantala is an ideal candidate as a benchmark system, given the wide set of experimental, e.g., Refs. [47-56], and numerical [36,57] studies.

The paper is organized as follows. Section II illustrates the tensile mechanical spectroscopy. The model and the numerical methods are given in Sec. III. Results are presented and discussed in Sec. IV and summarized in Sec. V.

\section{TENSILE MECHANICAL SPECTROSCOPY}

Our main objective is to scrutinise the friction losses and assess the validity of Eq. (3). Friction losses become apparent at low frequency below $\sim 0.1 \mathrm{THz}$ where both Rayleigh and AD mechanisms are negligible with respect to them $[18,20]$. TED losses need special consideration. Like in AD, they drop at low frequency steeply, i.e., as $1 / Q_{\mathrm{TED}}(f) \propto f$ provided that $f \ll 1 / \tau_{\mathrm{TED}}$. The magnitude of $\tau_{\mathrm{TED}}$ is a delicate matter. In fact, TED is due to both longitudinal and transverse thermal currents following longitudinal and transverse, e.g., flexural, vibrations. If TED follows from transverse currents, $\tau_{\mathrm{TED}}$ depends on the shape of the specimen and is proportional to its cross section [22,25]. The influence of extrinsic features makes a numerical approach at atomic length scale debatable. Instead, the drawback is not present for longitudinal currents (a)
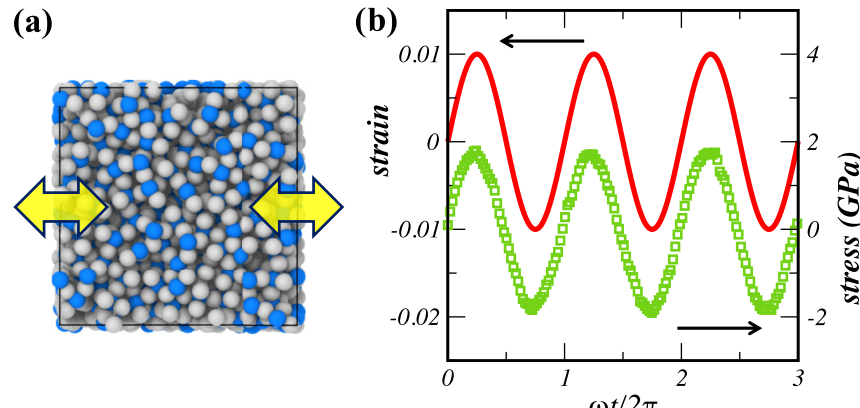

FIG. 1. (a) Tensile mechanical spectroscopy of tantala glass. (b) Representative plots of the applied oscillatory strain $\left(\omega=10^{12} \mathrm{rad} / \mathrm{s}\right)$ and the resulting stress.

where only intrinsic properties are involved [22,25]

$$
\tau_{L-\mathrm{TED}}=\frac{\kappa}{v^{2} C_{V}},
$$

where $\kappa, C_{V}$, and $v$ are the thermal conductivity, the specific heat per unit volume, and the velocity of sound, respectively. For tantala $\tau_{L-\mathrm{TED}} \simeq 1.7 \mathrm{ps}\left(\kappa=33 \mathrm{Wm}^{-1} \mathrm{~K}^{-1}\right.$ and $C_{V}=$ $2.1 \times 10^{6} \mathrm{~J} \mathrm{~m}^{-3} \mathrm{~K}^{-1}$ [58] and the approximate value of $v=$ $3 \times 10^{3} \mathrm{~m} / \mathrm{s}$ [59]). Since $\tau_{L-\mathrm{TED}}$ is comparable to $\tau_{A D}[18,20]$ and TED and AD have the same frequency scaling at low frequency, see Eq. (2), one expects that, if TED is induced by longitudinal waves only is, such as $\mathrm{AD}$, negligible with respect to TARP mechanism below $\sim 0.1 \mathrm{THz}[18,20]$.

The previous remarks prompted us to design an in silico mechanical spectroscopy with the aim at exciting only longitudinal vibrations. More explicitly, we performed nonequilibrium molecular-dynamics (NEMD) simulations of glassy tantala created from the melt with different cooling rates. Following the protocol of mechanical spectroscopy adopted in experiments [60-62] and simulations [46,63-66], the glass is subjected to periodic tensile elongation along one single direction perpendicular to a face with frequency $f$, and the storage and the dissipative parts of the dynamic elastic modulus, $E^{\prime}(f)$ and $E^{\prime \prime}(f)$, respectively, are evaluated in the linearresponse regime $(500 \mathrm{MHz} \leqslant f \leqslant 1 \mathrm{THz})$, see Fig. 1. Apart from the elongation direction, no deformation occurs in other directions. The quality factor is determined by the ratio,

$$
Q(f)=\frac{E^{\prime}(f)}{E^{\prime \prime}(f)} .
$$

Having set the deformation scheme, $Q(f)$ depends only on the adopted microscopic force field with no other assumptions.

\section{MODEL AND METHODS}

Classical molecular-dynamics simulations for amorphous tantala were carried out using LAMMPS software [67]. We model tantala $\left(\mathrm{Ta}_{2} \mathrm{O}_{5}\right)$ using a modified van Beest, Kramer, and van Santen potential [68] with an additional pseudocovalent Morse term [69]. For computational reasons, we implemented Wolf truncation with a cutoff function as proposed in Ref. [57]. Each simulation consists of 2520 atoms, contained in a cubic box with periodic boundary conditions. Glassy samples were produced cooling high-temperature liquids. Tantala 
crystal is first equilibrated at $300 \mathrm{~K}$ and then rapidly heated to $5000 \mathrm{~K}$. The liquid at $5000 \mathrm{~K}$ is equilibrated for $50 \mathrm{~ns}$ and then cooled down to $0 \mathrm{~K}$ at constant quench rate with an $N-P-T$ ensemble. During the quench run, configurations at the temperatures of interest were collected, equilibrated again for $50 \mathrm{ps}$ and finally energy minimized. With this protocol, we generated 30 independent samples, whose amorphous structure is confirmed after examining their pair distribution function. The numerical analog of mechanical spectroscopy is achieved by imposing to the simulation box a sinusoidal tensile strain $\epsilon_{i i}(t)=\epsilon_{0} \sin (\omega t)$ in the $i$ direction as sketched in Fig. 1(a) and measuring the corresponding tensile stress along the same direction $\sigma_{i i}$. No deformation of the simulation box takes place in other directions. The results are averaged over all three directions $(i=X, Y$, or $Z)$. The frequency $f=\omega / 2 \pi$ is varied from $0.5 \mathrm{GHz}$ to $1 \mathrm{THz}$. The frequency range is below the Ioffe-Regel limit, i.e., below a few terahertz and $Q>\pi$ where the wavelength of the sound waves with attenuation factor $\Gamma=\omega / Q$ is larger than the average interparticle distance and the notion of a phonon with a welldefined wave vector is applicable [17]. We fix the amplitude $\epsilon_{0}=0.01$ such that the deformation is in the linear elastic regime. In order to maintain constant temperature conditions and dissipate the heat produced during the deformation, a Berendsen thermostat is employed. As a result of deformation, the corresponding component of the internal stress $\Sigma(t)$ has an oscillatory behavior at the same frequency of the strain as shown in Fig. 1(b). Mechanical dissipation $Q^{-1}$ is derived via the relation,

$$
Q^{-1}=\left[\int_{0}^{N_{c} T_{\omega}} \cos (\omega t) \Sigma(t) d t\right] /\left[\int_{0}^{N_{c} T_{\omega}} \sin (\omega t) \Sigma(t) d t\right]
$$

where $T_{\omega}=f^{-1}$ is the oscillation period and $N_{c}$ is the number of strain cycles. In the following, $Q^{-1}$ is calculated during, at least, 20 deformation cycles, i.e., $N_{c} \geqslant 20$.

\section{RESULTS AND DISCUSSION}

First, we focus on the glass preparation via melt cooling. Figure 2(a) shows the temperature dependence of the specific volume at different cooling rates. The knee signals the vitrification of the sample. One observes better densification at lower cooling rates and final densities at $300 \mathrm{~K}$ well in the experimental range of the amorphous solids prepared by deposition $[55,56]$. Figure 2(b) shows that the fictive temperature, i.e., the position of the knee, decreases with the logarithm of the cooling rate in agreement with the experiments [70]. Figure 2(c) shows that the slower the cooling rate the lower the inherent energy of the glass, i.e., the energy deprived of the thermal energy, thus, suggesting an exploration of the deeper states of the energy landscape [71].

Figure 3 illustrates the frequency dependence of the storage (top) and the dissipative (bottom) parts of the dynamic elastic modulus of a tantala glass. Below $\sim 0.2 \mathrm{THz}$, the larger magnitude of $E^{\prime}$ with respect to $E^{\prime \prime}$ evidences the limited mechanical losses which increase at higher temperatures where the ratio $E^{\prime} / E^{\prime \prime}$ becomes smaller. Figure 3(a) shows that the frequency dependence of the storage modu-
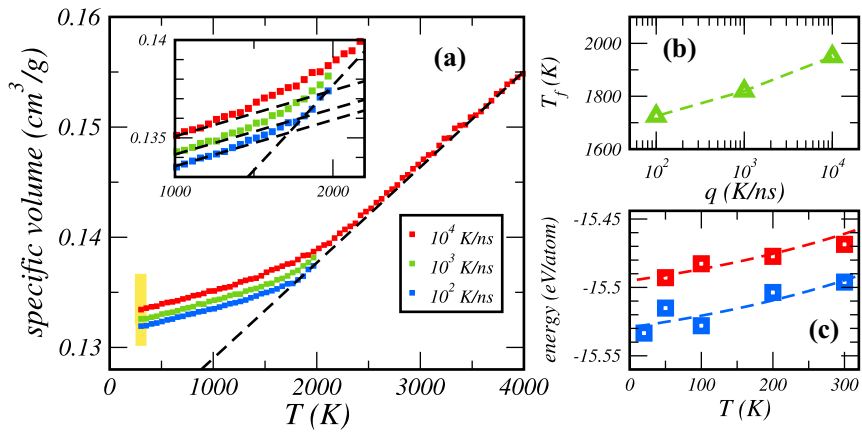

FIG. 2. (a) Temperature dependence of the specific volume at different cooling rates. The yellow bar signals the range of the experimental values of the amorphous films $[55,56]$. The inset is a magnification of the knee defining the fictive temperature. (b) The quasilogarithmic decrease in the fictive temperature with the cooling rate. (c) Inherent energy of the glass in the low-temperature range [the same symbols as in panel (a)]. The dashed lines are guides for the eyes.

lus is not monotonous. On increasing the frequency below $\sim 0.2 \mathrm{THz}, E^{\prime}(f)$ slightly increases. At higher temperatures, the increase is more pronounced. Above $\sim 0.2 \mathrm{THz}$, a sharp drop is observed. On the other hand, the dissipative part of the dynamical modulus $E^{\prime \prime}(f)$ increases mildly with the frequency below $\sim 0.2 \mathrm{THz}$ with a much steeper growth at higher frequencies, see Fig. 3(b). The joint increase in both $E^{\prime}(f)$ and $E^{\prime \prime}(f)$ for $f \lesssim 100 \mathrm{GHz}$ is characteristic of disordered solids at low frequencies [29]. The nature of the rapid changes in both $E^{\prime}(f)$ and $E^{\prime \prime}(f)$ for $f \gtrsim 100 \mathrm{GHz}$ will become clear by discussing their ratio, i.e., the quality factor Eq. (5) in the next paragraph.

Figure 4 reports the main results of the mechanical spectroscopy analysis, i.e., the frequency dependence of the inverse quality factor $Q$ under isothermal conditions following different cooling rates. Figures 4(a) and 4(b) show that at high frequencies $(f \sim 1 \mathrm{THz})$ the losses change according to a temperature-independent power-law $Q \propto f^{-3}$, consistent with the Rayleigh scattering [17,19-21]. This provides an explanation of the steep changes in the dynamic modulus observed at high frequency in Fig. 3. At lower frequency, after a crossover region between approximately 100 and $300 \mathrm{GHz}$,
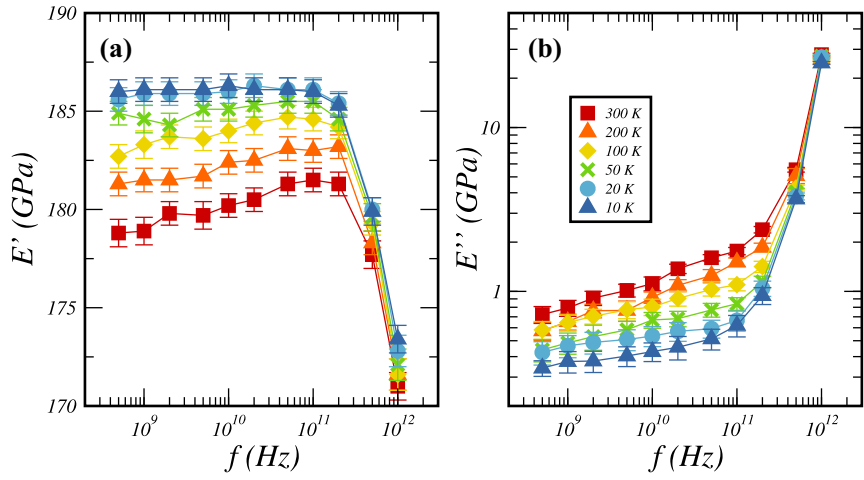

FIG. 3. (a) Storage and (b) loss components of the dynamic elastic modulus. 

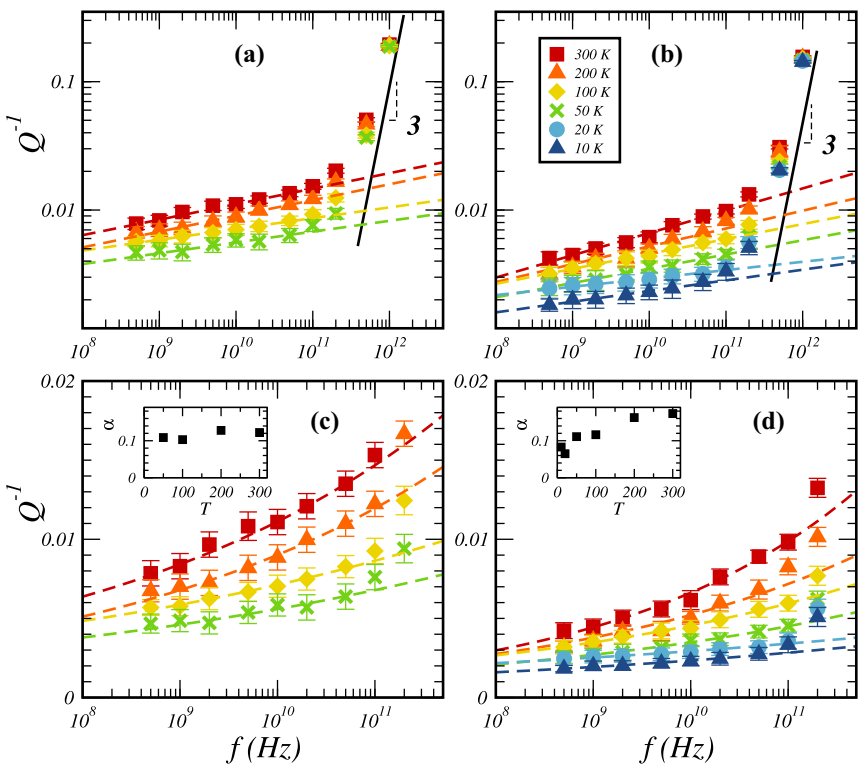

FIG. 4. Isothermal frequency dependence of the inverse quality factor $Q^{-1}$ for the glasses cooled at (a) $10^{4} \mathrm{~K} / \mathrm{ns}$ and (b) $10^{2} \mathrm{~K} / \mathrm{ns}$. The dashed lines are the best-fit curves with Eq. (7). At high frequencies $(f \sim 1 \mathrm{THz}) Q \propto f^{-3}$ consistent with the Rayleigh scattering [17,19-21]. At lower frequency, after a crossover region, a well-defined temperature-dependent power-law regime is observed. Panels (c) and (d) magnify in lin-log plots the power-law regimes of the panels (a) and (b), respectively. The insets: exponent $\alpha$ according to Eq. (7). The temperature dependence of the exponent vanishes by increasing the cooling rate.

where $1 / Q$ changes in frequency with an effective slope between about 0.2 and 3 in the log-log plot, one systematically observes a well-defined frequency power-law regime,

$$
Q(f) \propto f^{-\alpha},
$$

with $\alpha \sim 0.1$ to 0.2 , depending on both temperature and cooling rate. The frequency dependence of $1 / Q(f)$ below $100 \mathrm{GHz}$ is not consistent with both $\mathrm{AD}$ and TED. In particular, for frequencies lower than $1 / \tau_{L-\mathrm{TED}} \sim 1 / \tau_{A D} \sim 1 \mathrm{THz}$, see Sec. II, their contribution to $1 / Q(f)$ is proportional to frequency, see Eq. (2), i.e., much steeper than the observed one. Our simulations suggest that, if AD and TED have significant magnitude with respect to the other damping mechanisms, their contribution is limited to the crossover region between about 100 and $300 \mathrm{GHz}$ and only internal friction is effective at lower frequency. Figures 4(c) and 4(d) magnify in lin-log plots the power-law regimes. We do not observe deviations from Eq. (7) down to $f \sim 500 \mathrm{MHz}$. The systematic presence of a power-law frequency dependence of the quality factor at low frequency is supported by a large body of evidence. For a recent broad overview, see Ref. [27] and more specific studies concerning sediment, soil and rocks [72], random media [73], porous structures [74], amorphous silica [21], unstressed silicon nitride [75], other anelastic solids [28,29,76,77], as well as biological tissues [78]. Even if ubiquitous, the interpretation of Eq. (7) is not unique. The quality factor is related to the force spectral density via $Q(f) \propto 1 / f S(f)$ [15], and then it is natural to read Eq. (7) as manifestation of the low-frequency $1 / f$ noise $[79,80]$. In particular, Eq. (7) is recovered in multi-

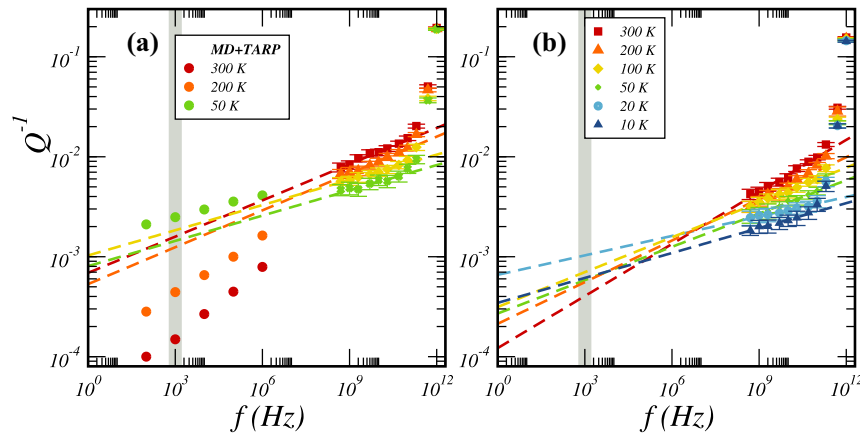

FIG. 5. Low-frequency extrapolation of the power-law regime of the isothermal inverse quality factor $Q^{-1}$ for the glasses cooled at (a) $10^{4} \mathrm{~K} / \mathrm{ns}$ and (b) $10^{2} \mathrm{~K} / \mathrm{ns}$. The same color code and symbols as in Fig. 4. The fast-quenched sample exhibits a quality factor at low frequency, e.g., $1 \mathrm{kHz}$, which is nearly temperature independent, different from the slowly quenched sample. For comparison, panel (a) includes also MD-TARP data concerning a glass made by cooling a melt at $3.5 \times 10^{4} \mathrm{~K} / \mathrm{ns}$ [36]. Note their much stronger temperature dependence.

plicative schemes where relaxation depends on the successful outcome of several independent steps [80-82]. Equation (7) may be also understood in the framework of additive schemes as the weighted sum over a set of independent dissipation entities characterized by a broad range of thermally activated relaxation rates due to a nearly flat distribution of energy barriers [79], i.e., the view adopted by the TARP scheme. Within the additive scheme, Eq. (7) with $0<\alpha<1$ is recovered by resorting to the universal features of the statistics of extremely low-energy states [83] leading to the exponential distribution of the energy barriers $p(V)=p_{\exp }(V) \propto \exp \left[-V / k_{B} T_{0}\right][84]$ where $T_{0}$ is an effective temperature, yielding $\alpha=T / T_{0}$ at a given temperature $T[28,76,84,85]$. Our simulations show that the temperature dependence of the $\alpha$ exponent in Eq. (7) is fairly weak and increases for glasses prepared with smaller cooling rates, see Fig. 4, insets of panels (c) and (d), which are compatible with a narrowing of $p(V)$.

Figure 5 is plotted arguing the validity of the power-law regime of the frequency dependence of the isothermal quality factor down to very low frequencies. In particular, Fig. 5(a) compares the loss of the glass cooled at $q=10^{4} \mathrm{~K} / \mathrm{ns}$ with the predictions of the TARP model Eq. (3) with parameters drawn by $\mathrm{MD}$ simulations of an identical $\mathrm{Ta}_{2} \mathrm{O}_{5}$ glass model, prepared by cooling the melt at a comparable rate $q=3.5 \times 10^{4} \mathrm{~K} / \mathrm{ns}[36]$. It is seen that the MD-TARP model recovers the low-frequency power-law Eq. (7). However, by increasing the temperature above $50 \mathrm{~K}$, it predicts increasing smaller losses with respect to ours, which is not consistent with the larger cooling rate used to prepare the glass. Figure 5(b) shows the losses of the glass prepared by lower cooling rate $\left(q=10^{2} \mathrm{~K} / \mathrm{ns}\right)$ calculated by NEMD. With respect to the glass prepared with $q=10^{4} \mathrm{~K} / \mathrm{ns}$, they exhibit much stronger temperature dependence at low frequency. Unfortunately, no related MD-TARP predictions are reported.

To discriminate between the effectiveness of the NEMD approach and the MD-TARP model, we compared the two methods, both concerning with quench-cooled glasses, with measurements concerning amorphous films created by 

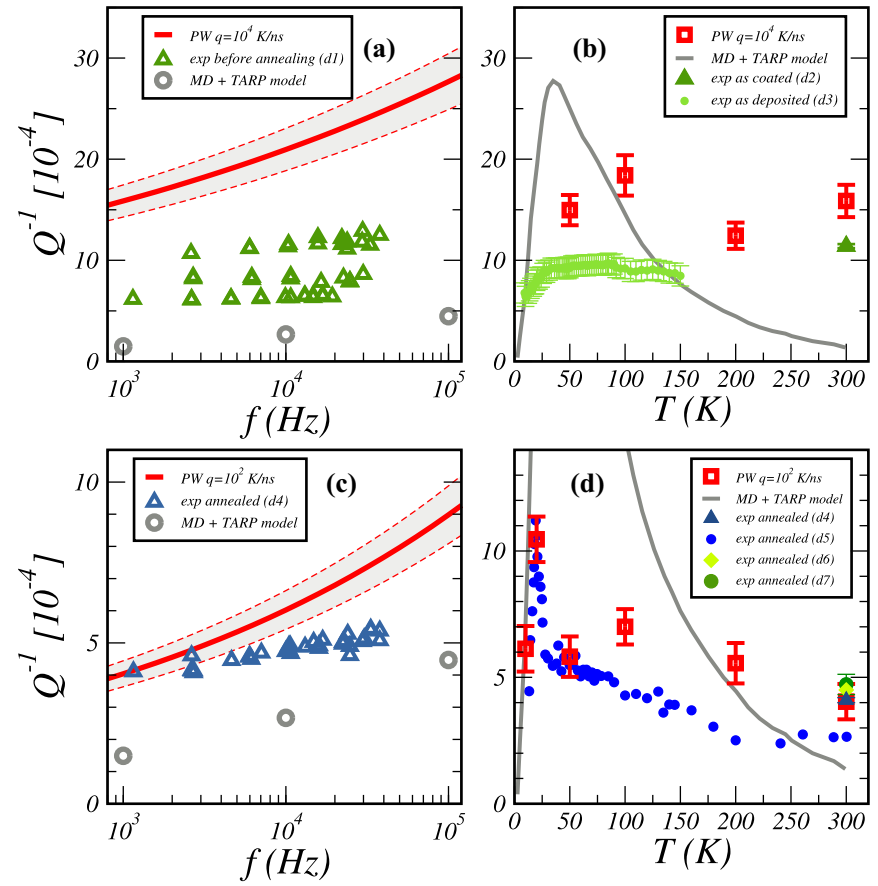

FIG. 6. Frequency (left) and temperature (right) dependence of the inverse quality factor $Q^{-1}$ at $300 \mathrm{~K}$ and $f=1 \mathrm{kHz}$, respectively, of the glasses produced by quench cooling at $10^{4}$ (top) and $10^{2} \mathrm{~K} / \mathrm{ns}$ (bottom). MD-TARP data from Ref. [36]. Experimental data from Refs. [48,49] (d1), [50] (d2), [51] (d3), [48,49] (d4), [52] (d5), [53] (d6), and [54] (d7). The dashed lines in panels (a) and (c) bound the uncertainty of the NEMD data from the present paper (PW).

deposition [48-54]. This seemingly hazardous step was, in part, driven by the missing, to the best of our knowledge, mechanical data concerning quench-cooled glassy tantala but, it is also fully justified in view of recent experiments concluding that the amorphous tantala film shares a common progenitor state with a hypothetical glass quenched from the melt [47]. The results are summarized in Fig. 6. Panel (a) compares the low-frequency losses at $T=300 \mathrm{~K}$ of not annealed films prepared with different procedures $[48,49]$ with the ones evaluated for glasses prepared by fast quench [data from Fig. 4(c)]. The significant spread of the measurements results from the known influence of different sample production processes and makes difficult a comparison with the evaluated $Q$ values. However, a clearer picture is provided by measurements at different temperatures with $f=1 \mathrm{kHz}$ [50,51]. In fact, Fig. 6(b) shows that, although the losses evaluated by the MD-TARP model for the glass cooled with rate $q=3.5 \times 10^{4} \mathrm{~K} / \mathrm{ns}$ [36] are inconsistent with the experiment, our NEMD data for the glass with $q=10^{4} \mathrm{~K} / \mathrm{ns}$ deviate from the experiment not more than $\sim 50 \%$, capturing the weak role of the temperature. In a further effort, we profited from the fact that suitable annealing yields mechanical losses much less dependent on the deposition procedure [48,49,52-54]. Figures 6(c) and 6(d) compare the measurements concerning annealed amorphous tantala films [48,49,52-54] with the NEMD results concerning a glass prepared by slower melt cooling $\left(q=10^{2} \mathrm{~K} / \mathrm{ns}\right)$. The agreement is remarkable, giving strong support to the possibility to match the properties of disordered structures created by extremely different routes. For completeness, we also plot in Figs. 6(c) and 6(d) the MD-TARP predictions concerning a glass made by $q=3.5 \times 10^{4} \mathrm{~K} / \mathrm{ns}$ shown in Figs. 6(a) and 6(b) (the only cooling rate studied in Ref. [36]). Again, large disagreement is seen, especially in the temperature dependence of the loss which is much stronger than the experimental one. The predictions of the TARP model cannot improve by using as input data to Eq. (3) the ones of the glass we used in our NEMD and made by cooling at $q=$ $10^{2} \mathrm{~K} / \mathrm{ns}$. In fact, the comparison of Figs. 4(c) and 4(d) shows that the lower the cooling rate, the stronger the temperature dependence of the losses. This follows by the narrowing of the energy barrier distribution due to the better annealing of the glass and suggests that the TARP model would predict losses with much stronger temperature dependence than using the data of the glass made with $q=3.5 \times 10^{4} \mathrm{~K} / \mathrm{ns}$, the latter dependence already being stronger than the experimental one.

\section{CONCLUSIONS}

We have reported a numerical approach, covering by MD simulations about 3.3 decades in frequency $(500 \mathrm{MHz} \leqslant f \leqslant$ $1 \mathrm{THz}$ ), which, combined with an informed extrapolation scheme, allows one to evaluate by an agnostic approach the mechanical loss in disordered solids over about nine decades in frequency (kilohertz to terahertz). We demonstrate our approach in tantala evidencing remarkable agreement with the available experimental data concerning annealed amorphous films created by deposition in the temperature range spanning from cryogenic to room values $(10-300 \mathrm{~K})$, in sharp contrast with the poor accuracy of the TARP scheme. The latter finding points to the possibility to interpret the mechanical properties of amorphous annealed film in terms of a glassy state produced by properly cooling from the melt. Our results, capturing weakly frequency-dependent low losses in a quantitative way, suggest that in silico mechanical spectroscopy has the potential to push its predictive power down to the millisecond timescale and to rationalize in a powerful way mechanical losses of amorphous materials that are critically important for nanosystems and gravitational wave detection [11-14]. We anticipate that our approach could be extended to other materials, including glasses exhibiting reduced friction in lowfrequency and room-temperature conditions. This is the case of $\mathrm{SiO}_{2}$ [26] where, notably, the power-law regime Eq. (7) sets in below $\sim 300 \mathrm{GHz}$ [21], thus, resulting in an increased overlap with simulation frequencies with respect to tantala and ensuring a reliable extrapolation down to low frequencies.

\section{ACKNOWLEDGMENT}

A generous grant of computing time from IT Center, University of Pisa and Dell® Italia is gratefully acknowledged.
[1] M. Aspelmeyer, T. J. Kippenberg, and F. Marquardt, Rev. Mod. Phys. 86, 1391 (2014).
[2] T. Ramos, V. Sudhir, K. Stannigel, P. Zoller, and T. J. Kippenberg, Phys. Rev. Lett. 110, 193602 (2013). 
[3] M. Eichenfield, R. Camacho, J. Chan, K. J. Vahala, and O. Painter, Nature (London) 459, 550 (2009).

[4] R. Leijssen, G. R. La Gala, L. Freisem, J. T. Muhonen, and E. Verhagen, Nat. Commun. 8, 16024 (2017).

[5] T. B. Gabrielson, IEEE Trans. Electron Devices 40, 903 (1993).

[6] M. Li, H. X. Tang, and M. L. Roukes, Nat. Nanotechnol. 2, 114 (2007).

[7] S. Schmid, L. G. Villanueva, and M. L. Roukes, Fundamentals of Nanomechanical Resonators (Springer, Berlin, 2016).

[8] T. Bagci, A. Simonsen, S. Schmid, L. G. Villanueva, E. Zeuthen, J. Appel, J. M. Taylor, A. Sørensen, K. Usami, A. Schliesser et al., Nature (London) 507, 81 (2014).

[9] Y. Y. Jiang, A. D. Ludlow, N. D. Lemke, R. W. Fox, J. A. Sherman, L. S. Ma, and C. W. Oates, Nat. Photon. 5, 158 (2011).

[10] R. P. Leland, IEEE Sens. J. 5, 493 (2005).

[11] M. Pitkin, S. Reid, S. Rowan, and J. Hough, Liv. Rev. Relativ. 14, 5 (2011).

[12] G. M. Harry (LIGO Scientific Collaboration), Class. Quantum Grav. 27, 084006 (2010).

[13] T. Akutsu (KAGRA Collaboration), J. Phys.: Conf. Ser. 610, 012016 (2015).

[14] F. Acernese, M. Agathos, K. Agatsuma, D. Aisa, N. Allemandou, A. Allocca, J. Amarni, P. Astone, G. Balestri, G. Ballardin et al., Class. Quantum Grav. 32, 024001 (2014).

[15] P. R. Saulson, Phys. Rev. D 42, 2437 (1990).

[16] J. Jäckle, L. Piché, W. Arnold, and S. Hunklinger, J. Non-Cryst. Solids 20, 365 (1976).

[17] B. Rufflé, G. Guimbretière, E. Courtens, R. Vacher, and G. Monaco, Phys. Rev. Lett. 96, 045502 (2006).

[18] E. Rat, M. Foret, G. Massiera, R. Vialla, M. Arai, R. Vacher, and E. Courtens, Phys. Rev. B 72, 214204 (2005).

[19] G. Baldi, V. M. Giordano, G. Monaco, and B. Ruta, Phys. Rev. Lett. 104, 195501 (2010).

[20] C. Ferrante, E. Pontecorvo, G. Cerullo, A. Chiasera, G. Ruocco, W. Schirmacher, and T. Scopigno, Nat. Commun. 4, 1793 (2013).

[21] A. Huynh, E. Péronne, C. Gingreau, X. Lafosse, A. Lemaître, B. Perrin, R. Vacher, B. Rufflé, and M. Foret, Phys. Rev. B 96, 174206 (2017).

[22] A. Nowick and B. Berry, Anelastic Relaxation in Crystalline Solids (Academic Press, New York, 1972).

[23] H. J. Maris, Phys. Acoust. 8, 279 (1971).

[24] R. Vacher, E. Courtens, and M. Foret, Phys. Rev. B 72, 214205 (2005).

[25] C. Zener, Proc. Phys. Soc. 52, 152 (1940).

[26] K. A. Topp and D. G. Cahill, Z. Phys. B: Condens. Matter 101, 235 (1996).

[27] S. Holm, Waves with Power-Law Attenuation (Springer, Cham, Switzerland, 2019).

[28] R. L. Bagley and J. Torvik, AIAA J. 21, 741 (1983).

[29] T. Pritz, Appl. Acoust. 65, 1027 (2004)

[30] H. Kolsky, Philos. Mag. 1, 693 (1956).

[31] E. Kjartansson, J. Geophys. Res.: Solid Earth 84, 4737 (1979).

[32] O. Arcizet, V. Jacques, A. Siria, P. Poncharal, P. Vincent, and S. Seidelin, Nat. Phys. 7, 879 (2011).

[33] S. Kolkowitz, A. C. Bleszynski Jayich, Q. P. Unterreithmeier, S. D. Bennett, P. Rabl, J. G. E. Harris, and M. D. Lukin, Science 335, 1603 (2012).
[34] M. Hamoumi, P. E. Allain, W. Hease, E. Gil-Santos, L. Morgenroth, B. Gérard, A. Lemaître, G. Leo, and I. Favero, Phys. Rev. Lett. 120, 223601 (2018).

[35] K. S. Gilroy and W. A. Phillips, Philos. Mag. B 43, 735 (1981).

[36] J. P. Trinastic, R. Hamdan, C. Billman, and H.-P. Cheng, Phys. Rev. B 93, 014105 (2016).

[37] M. I. Klinger, Glassy Disordered Systems (World Scientific, Singapore, 2013).

[38] C. C. Yu and A. J. Leggett, Comments Condens. Matter Phys. 14, 231 (1988).

[39] A.L.Burin, D. Natelson, D. Osheroff, and Y. Kagan, in Tunneling Systems in Amorphous and Crystalline Solids, edited by $\mathrm{P}$ Esquinazi (Springer, Berlin, 1998), Chap. 5, pp. 223-315.

[40] T. Pérez-Castañeda, C. Rodríguez-Tinoco, J. Rodríguez-Viejo, and M. A. Ramos, Proc. Natl. Acad. Sci. USA 111, 11275 (2014).

[41] A. Tanguy, J. P. Wittmer, F. Leonforte, and J.-L. Barrat, Phys. Rev. B 66, 174205 (2002).

[42] A. Lemaître and C. Maloney, J. Stat. Phys. 123, 415 (2006).

[43] F. Puosi, J. Rottler, and J.-L. Barrat, Phys. Rev. E 89, 042302 (2014).

[44] A. Giuntoli and D. Leporini, Phys. Rev. Lett. 121, 185502 (2018).

[45] R. O. Pohl, X. Liu, and E. Thompson, Rev. Mod. Phys. 74, 991 (2002).

[46] T. Damart, A. Tanguy, and D. Rodney, Phys. Rev. B 95, 054203 (2017).

[47] O. L. G. Alderman, C. J. Benmore, J. Neuefeind, E. Coillet, A. Mermet, V. Martinez, A. Tamalonis, and R. Weber, Phys. Rev. Mater. 2, 043602 (2018).

[48] A. Amato, G. Cagnoli, M. Canepa, E. Coillet, J. Degallaix, V. Dolique, D. Forest, M. Granata, V. Martinez, C. Michel et al., J. Phys.: Conf. Ser. 957, 012006 (2018).

[49] M. Granata, A. Amato, L. Balzarini, M. Canepa, J. Degallaix, D. Forest, V. Dolique, L. Mereni, C. Michel, L. Pinard, B. Sassolas, J. Teillon, and G. Cagnoli, arXiv:1909.03737.

[50] M. Granata, E. Saracco, N. Morgado, A. Cajgfinger, G. Cagnoli, J. Degallaix, V. Dolique, D. Forest, J. Franc et al., Phys. Rev. D 93, 012007 (2016).

[51] R. Robie, Ph.D. thesis, University of Glasgow, School of Physics and Astronomy, 2018.

[52] I. W. Martin, R. Bassiri, R. Nawrodt, M. M. Fejer, A. Gretarsson, E. Gustafson, G. Harry, J. Hough, I. MacLaren, S. Penn et al., Class. Quantum Grav. 27, 225020 (2010).

[53] G. Vajente, R. Birney, A. Ananyeva, S. Angelova, R. Asselin, B. Baloukas, R. Bassiri, G. Billingsley, M. M. Fejer, D. Gibson et al., Class. Quantum Grav. 35, 075001 (2018).

[54] M. Principe, I. M. Pinto, V. Pierro, R. DeSalvo, I. Taurasi, A. E. Villar, E. D. Black, K. G. Libbrecht, C. Michel, N. Morgado et al., Phys. Rev. D 91, 022005 (2015).

[55] R. Bassiri, K. Evans, K. Borisenko, M. Fejer, J. Hough, I. MacLaren, I. Martin, R. Route, and S. Rowan, Acta Mater. 61, 1070 (2013).

[56] T. Li, F. A. Aguilar Sandoval, M. Geitner, L. Bellon, G. Cagnoli, J. Degallaix, V. Dolique, R. Flaminio, D. Forest, M. Granata et al., Phys. Rev. D 89, 092004 (2014).

[57] T. Damart, E. Coillet, A. Tanguy, and D. Rodney, J. Appl. Phys. 119, 175106 (2016).

[58] L. Ju, G. Harry, and B. Lee, in Advanced Gravitational Wave Detectors, edited by D. G. Blair, E. J. Howell, L. Ju, and 
C. Zhao (Cambridge University Press, Cambridge, UK, 2012), pp. 186-201.

[59] T. J. Vogler, S. Root, M. D. Knudson, and W. D. Reinhart, Sandia National Laboratories Technical Report No. SAND20116770, 2011 (unpublished).

[60] J. Qiao, J.-M. Pelletier, and R. Casalini, J. Phys. Chem. B 117, 13658 (2013).

[61] C. Liu, E. Pineda, and D. Crespo, Metals 5, 1073 (2015).

[62] M. Rubinstein and R. H. Colby, Polymer Physics (Oxford University Press, Oxford, 2003).

[63] M. Vladkov and J.-L. Barrat, Macromol. Theory Simul. 15, 252 (2006).

[64] B. Shang, J. Rottler, P. Guan, and J.-L. Barrat, Phys. Rev. Lett. 122, 105501 (2019).

[65] J. Wittmer, H. Xu, O. Benzerara, and J. Baschnagel, Mol. Phys. 113, 2881 (2015).

[66] V. V. Palyulin, C. Ness, R. Milkus, R. M. Elder, T. W. Sirk, and A. Zaccone, Soft Matter 14, 8475 (2018).

[67] S. Plimpton, J. Comput. Phys. 117, 1 (1995).

[68] B. W. H. van Beest, G. J. Kramer, and R. A. van Santen, Phys. Rev. Lett. 64, 1955 (1990).

[69] J. P. Trinastic, R. Hamdan, Y. Wu, L. Zhang, and H.-P. Cheng, J. Chem. Phys. 139, 154506 (2013).

[70] J. Jäckle, Rep. Prog. Phys. 49, 171 (1986).
[71] P. G. Debenedetti and F. H. Stillinger, Nature (London) 410, 259 (2001).

[72] K. Aki and P. G. Richards, Quantitative Seismology, 2nd ed. (University Science Books, Sausalito, CA, 2002).

[73] J. Garnier and K. Sølna, Waves Random Complex Media 25, 482 (2015).

[74] S. R. Pride and Y. J. Masson, Phys. Rev. Lett. 97, 184301 (2006).

[75] Q. P. Unterreithmeier, T. Faust, and J. P. Kotthaus, Phys. Rev. Lett. 105, 027205 (2010).

[76] M. Caputo and F. Mainardi, Riv. Nuovo Cimento Soc. Ital. Fis. 1, 161 (1971).

[77] T. L. Szabo, J. Acoust. Soc. Am. 96, 491 (1994).

[78] R. Sinkus, K. Siegmann, T. Xydeas, M. Tanter, C. Claussen, and M. Fink, Magn. Reson. Med. 58, 1135 (2007).

[79] M. B. Weissman, Rev. Mod. Phys. 60, 537 (1988).

[80] A. Amir, Y. Oreg, and Y. Imry, Proc. Natl. Acad. Sci. USA 109, 1850 (2012).

[81] E. W. Montroll and M. F. Shlesinger, Proc. Nat. Acad. Sci. USA 79, 3380 (1982).

[82] L. A. Dissado and R. M. Hill, Proc. R. Soc. London Ser. A 390, 131 (1983).

[83] J.-P. Bouchaud and M. Mézard, J. Phys. A 30, 7997 (1997).

[84] J. P. Bouchaud, J. Phys. I France 2, 1705 (1992).

[85] W. Chen and S. Holm, J. Acoust. Soc. Am. 115, 1424 (2004) 